\title{
Systemic Sarcoidosis Associated to IgA Nephropathy
}

\author{
Madiha Mahfoudhi*, Imen Gorsane, Mounira El Euch, Rym Goucha, Sami Turki, \\ Taieb Ben Abdallah \\ Internal Medicine A Department, Charles Nicolle Hospital, Tunis, Tunisia \\ Email: ${ }^{*}$ madiha mahfoudhi@yahoo.fr
}

Received 27 June 2015; accepted 18 August 2015; published 21 August 2015

Copyright (C 2015 by authors and Scientific Research Publishing Inc.

This work is licensed under the Creative Commons Attribution International License (CC BY).

http://creativecommons.org/licenses/by/4.0/

c) (i) Open Access

\begin{abstract}
The sarcoidosis was rarely associated to IgA nephropathy. We report a 38-year-old man presented decreased visual acuity and xerostomia. He had two axillary lymphadenopathies and pitting edema of legs in physical examination. The ophthalmological examination revealed a right posterior uveitis. Biological investigations showed a mild renal insufficiency and elevated serum level of angiotensin-converting enzyme, $\beta 2$ microglobulin and IgA. He had a proteinuria and a microscopic hematuria. The kidney echography was without abnormalities. Histological study of the renal biopsy found results in favor to IgA nephropathy. Biopsies performed in accessory salivary gland and lymph nodes revealed non-necrotising epitheloid and gigantocellular granulomatous inflammation suggesting a sarcoidosis. The diagnosis of a sarcoidosis associated to IgA nephropathy was posed. The treatment was based on oral prednisolone with gradual tapering doses. He regained normal vision. The renal function had not worsened. No relapse of sarcoidosis was noted during our follow up.
\end{abstract}

\section{Keywords}

Sarcoidosis, Granuloma, Proteinuria, IgA Nephropathy

\section{Introduction}

Sarcoidosis is a systemic disease of non-elucidated etiopathogenesis [1]. The essential lesion corresponds to epitheloid and gigantocellular granulomas without caseous necrosis located in various organs.

The lungs and the lymphatic systems are the most involved in this disease.

\footnotetext{
"Corresponding author.
}

How to cite this paper: Mahfoudhi, M., Gorsane, I., El Euch, M., Goucha, R., Turki, S. and Abdallah, T.B. (2015) Systemic Sarcoidosis Associated to IgA Nephropathy. Case Reports in Clinical Medicine, 4, 284-288. 
Sarcoidosis can be revealed by all forms of uveitis, neurological feature, interstitial pneumonitis, pulmonary fibrosis, dysrhythmia, cardiomyopathy, hypercalcemia, endocrine manifestations, sicca syndrome and renal disturbance [1].

Serum level of angiotensin-converting enzyme, synthesized by active macrophage, can be elevated. Serum $\beta 2$ microglobulin level can be also elevated signing lymphocyte activity. No specific diagnostic test is available for sarcoidosis, so it's still an exclusion diagnosis [1] [2].

The positive diagnosis is based on a set of clinical, biological, radiological and histological arguments after eliminating other infectious, systemic and drug granulomatous causes.

The most frequent signs suggesting sarcoidosis diagnosis are bilateral mediastinal lymphadenopathy or diffuse micronodular pulmonary infiltration at chest radiograph. Thoracic radiologic abnormalities are found in about $90 \%$ of patients being affected of sarcoidosis [2]-[4].

In half of cases, sarcoidosis resolves spontaneously within 2 years. Whereas refractory chronic forms of sarcoidosis are described leading to pulmonary and extrapulmonary fibrosis and organs impairment [4]-[6]. Multiple indicators of prognosis have been proposed [3].

A diagnosis difficulty is encountered in case of extrathoracic revelatory disturbance. The renal affection is rare and presents commonly as granulomatous interstitial nephritis or nephrocalcinosis and urinary lithiasis due to hypercalciuria [1] [2] [4].

Few cases of IgA nephropathy are published in association to sarcoidosis feature [7]-[9].

The aim of this study is to explain through this rare case and the literature if this association is fortuitous or has an etiopathogenic mechanism and determine the diagnosis difficulty and the prognosis profile.

\section{Case Report}

A 38-year-old man, nonsmoker, admitted for a recent decreased visual acuity and a xerostomia lasting for three months.

The physical examination showed normal body temperature and pulse rate. He had two right centimetric and painless axillary lymphadenopathies, a blood pressure of 140/90 mmHg and pitting edema of legs. The pulmonary, cardiac, abdominal, neurological, and skinny examinations were without abnormalities. The ophthalmological examination revealed a right posterior uveitis with a decreased visual acuity of 6/10. The electrocardiogram was without anomaly. Urine dipstick showed ++ of hematuria and +++ of proteinuria.

Biological investigations showed that blood cell counts, liver function tests, serum and urine calcium levels, serum electrolytes levels were within the normal range. A mild renal insufficiency with a creatinine of $170 \mu$ $\mathrm{mol} / \mathrm{l}$ was noted. Erythrocyte sedimentation rate was $40 \mathrm{~mm} / \mathrm{h}$. The proteinaemia was $50 \mathrm{~g} / \mathrm{l}$ and the serum albumin level was $27 \mathrm{~g} / \mathrm{l}$. Analysis of the urine found a proteinuria of $3.7 \mathrm{~g} /$ day and a microscopic hematuria. Thus, he had an impure nephrotic syndrome.

The serum angiotensin-converting enzyme level was 144 U/l. Serum $\beta 2$ microglobulin and IgA levels (720 $\mathrm{mg} / \mathrm{dl}$ ) were elevated.

Chest X ray and CT scan found no abnormalities especially mediastinal adenopathies or interstitial lesions. Full lung function tests, monooxide gas-transfer factor, bronchoscopy and bronchoalveolar lavage were normal.

The kidney echography was without abnormalities.

Histological study of the renal biopsy found proliferation of mesangial cells in the most of the glomeruli. There were no interstitial granulomatous infiltrates in all studied glomeruli. Immunohistochemistry showed important IgA and C3 deposits in the mesangium region corresponding to IgA nephropathy (Figure 1).

Anatomopathological examination of Biopsies performed in accessory salivary gland and lymph nodes revealed non-necrotising epitheloid and gigantocellular granulomatous inflammation suggesting a sarcoidosis.

Ziehl-Neelsen staining and Lowenstein culture were negative. Besides, tuberculin skin test and Quantiferon gold were negative.

The diagnosis of a sarcoidosis with ocular, ganglionnar and salivary involvement associated to IgA nephropathy was confirmed.

Corticosteroids with $1 \mathrm{mg} / \mathrm{Kg} / \mathrm{j}$ of oral prednisolone was initiated and gradually tapered over 6 months.

He regained normal vision. All clinical symptoms resolved. His serum angiotensin-converting enzyme level reached $18 \mathrm{U} / \mathrm{l}$. The renal function had not worsened. No relapse of proteinuria, hematuria or other location of sarcoidosis was noted during a follow up of four years. 


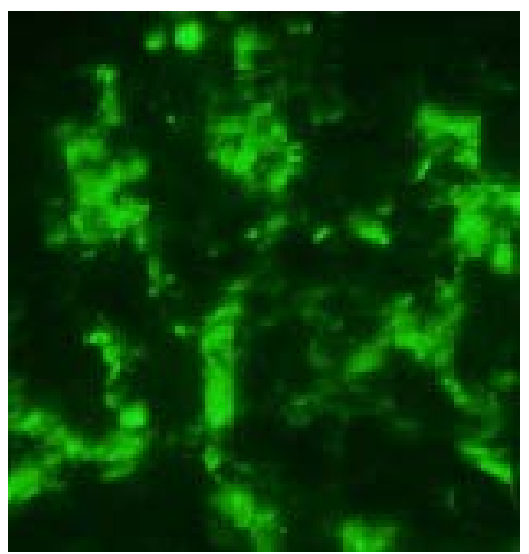

Figure 1. Immunohistochemistry of the renal biopsy: IgA mesangial deposits corresponding to IgA nephropathy.

\section{Discussion}

The sarcoidosis physiopathology is poorly understood. Genetic susceptibility and environmental factors may participate in sarcoidosis occurrence. Immunological exaggerated reactions to indefinite antigens with the presence of a particular genetic background induce the sarcoidosis granuloma formation [4]-[6].

The immune response implicates essentially macrophages, monocytes and CD4 lymphocytes.

The interaction between these actors causes the main systemic lesions of the sarcoidosis [4].

The kidney is rarely affected by the sarcoidosis. It manifests commonly as nephrocalcinosis secondary to hypercalcemia and non-caseating granulomatous interstitial nephritis [4].

Glomerulonephritis, exceptionally reported, included focul glomerulosclerosis, crescentic glomerulonephritis and IgA nephropathy [7].

Our patient had neither biological signs evoking interstitial nephritis nor radiological signs suggesting nephrocalcinosis or urinary lithiasis.

Before the performance of the biopsy (accessory salivary gland and adenopathy) in our case, the impure nephrotic syndrome, simultaneous to the other extrathoracic symptoms, induced us to think about a malignancy as a lymphoma complicated of a paraneoplastic extramembranous glomerulonephritis. The accessibility of biopsy sites facilitates the diagnosis. The clinical, biological and histological arguments with the absence of alternating disease were in favor of the sarcoidosis diagnosis. The kidney biopsy revealed unsuspected IgA nephropathy.

Many differential diagnosis corresponding to granulomatosis causes as several infections, drugs, vasculitis should be considered [1].

Tuberculosis is the main diagnosis which must be evoked in an endemic country. The research of Mycobacterium tuberculosis (Ziehl-Neelsen staining, Lowenstein culture, tuberculin skin test and Quantiferon gold) was negative in our patient eliminating a tuberculous origin. This step is determinant since a corticosteroid therapy indicated to an eventual sarcoidosis can worsen the prognosis if it is in fact tuberculosis [1] [4].

The absence of caseous necrosis is not a strong argument eliminating tuberculosis as necrosis may miss at the beginning of the disease.

Otherwise, sarcoidosis is characterized by anergy to tuberculin.

Few case reports concerning this rare association were published [8]-[11].

Vanhille, P. et al. reported the case of sarcoidosis complicated of acute renal failure due to rapidly progressive IgA glomerulonephritis [8].

Murray, F.E. et al. described a sarcoidosis and IgA nephropathy diagnosed simultaneously in a 23-year-old man [9].

The sarcoidosis can be revealed by the IgA nephropathy as reported by Tateno, S. et al. Their case concerned a 36 year old man, who had acute renal dysfunction due to IgA nephropathy and hypercalcemia. Sarcoidosis granulomas were revealed by muscle and skin biopsies [12].

Another case published by Nishiya, H. et al. illustrated the diagnosis difficulty in case of IgA nephropathy 
revealing sarcoidosis. Then, their patient was a 49-year-old woman presenting a feature mimicking a lymphoma. She had proteinuria, hematuria and nodular splenomegaly. The authors performed a splenectomy, and open renal biopsy with histological examination confirming the association of sarcoidosis and IgA nephropathy and excluding all malignancies [13].

Hamada, K. et al. described two cases of IgA nephropathy diagnosed during the clinical course of sarcoidosis which provide to them to emit the hypothesis of a common immunological background concerning these two diseases [14].

Our case had the peculiarity of having two serious complications, visual impairment which involves the functional prognosis and kidney disturbance that may engage the vital prognosis, hence there was a need for early diagnosis and rapid support to improve the general prognosis.

Through our present case and all these publications, we can suggest a physiopathological link between sarcoidosis and IgA nephropathy consisting of common immunological anomalies.

Some authors who published this association discussed if it's fortuitous or if the IgA nephropathy can be considered as an extrapulmonary manifestation of sarcoidosis.

Despite absence of physiological confirmation, clinicians must evoke possible association of IgA nephropathy to sarcoidosis [15].

Corticosteroids therapy is the conventional treatment prescribed in first intention in both cases of IgA nephropathy and sarcoidosis if there's an indication, but it has several adverse effect profile.

The clinical course of our patient was comparable of those reported in the literature.

The response is favorable after corticosteroid treatment. Taylor, J.E. et al. noted an improvement of renal function, an increase of albumin level, a lessening of proteinuria and hematuria with a decrease of calcium level [16].

Nishiki, M. et al. treated this association by oral administration of prednisone. The follow up was marked by the subsidence of cough and edema, the decrease of swelling hilar lymph nodes, pulmonary infiltrates and proteinuria, the normalization of serum angiotensin-converting enzyme and IgA levels. The dose was tapered without relapse occurrence [7].

The prognosis depends on the rapid diagnosis and the efficient treatment. IgA nephropathy associated to atypical extrathoracic signs of sarcoidosis can induce lateness in sarcoidosis diagnostic delay; leading to pulmonary fibrosis, myocarditis, severe neurological feature and worsening the prognosis. This association should be evoked by the clinician.

\section{Conclusion}

The sarcoidosis is rarely associated to IgA nephropathy. The diagnosis is based on the immunohistological resuls. An early diagnosis and treatment are necessary to avoid renal insufficiency.

\section{Disclosure Statement}

The authors have nothing to disclose.

\section{References}

[1] Iannuzzi, M.C. and Fontana, J.R. (2011) Sarcoidosis: Clinical Presentation, Immunopathogenesis, and Therapeutics. Journal of the American Medical Association, 305, 391-399. http://dx.doi.org/10.1001/jama.2011.10

[2] Criado, E., Sanchez, M., Ramirez, J., Arguis, P., de Caralt, T.M., Perea, R.J., et al. (2010) Pulmonary Sarcoidosis: Typical and Atypical Manifestations at High-Resolution CT with Pathologic Correlation. Radiographics, 30, 15671586. http://dx.doi.org/10.1148/rg.306105512

[3] Neville, E., Walker, A.N. and James, D.G. (1983) Prognostic Factors Predicting the Outcome of Sarcoidosis: An Analysis of 818 Patients. The Quarterly Journal of Medicine, 52, 525-533.

[4] Baughman, R.P., Lower, E.E. and du Bois, R.M. (2003) Sarcoidosis. The Lancet, 361, 1111-1118. http://dx.doi.org/10.1016/S0140-6736(03)12888-7

[5] Newman, L.S., Rose, C.S., Bresnitz, E.A., Rossman, M.D., Barnard, J., Frederick, M., et al. (2004) A Case Control Etiologic Study of Sarcoidosis: Environmental and Occupational Risk Factors. American Journal of Respiratory and Critical Care Medicine, 170, 1324-1330. http://dx.doi.org/10.1164/rccm.200402-249OC

[6] Muller-Quernheim, J., Schurmann, M., Hofmann, S., Gaede, K.I., Fischer, A., Prasse, A., et al. (2008) Genetics of 
Sarcoidosis. Clinics in Chest Medicine, 29, 391-414. http://dx.doi.org/10.1016/j.ccm.2008.03.007

[7] Nishiki, M., Murakami, Y., Yamane, Y. and Kato, Y. (1999) Steroid-Sensitive Nephrotic Syndrome, Sarcoidosis and Thyroiditis-A New Syndrome? Nephrology Dialysis Transplantation, 14, 2008-2010. http://dx.doi.org/10.1093/ndt/14.8.2008

[8] Vanhille, P., Beaudoin, D., Mougenot, B., Striker, L., Lemaître, V., Fleury, D., et al. (1986) Rapidly Progressing Glomerulonephritis with Mesangial IgA Deposits in Sarcoidosis. Nephrologie, 7, 207-209.

[9] Murray, F.E., Lombard, M.G., Donohoe, J.F., Doyle, G.D., Campbell, E. and Alton, B.G. (1987) Simultaneous Presentation of IgA Nephropathy and Sarcoidosis. Sarcoidosis, 4, 134-136.

[10] Chung-Park, M., Lam, M. and Yazdy, A.M. (1990) IgA Nephropathy Associated with Sarcoidosis. American Journal of Kidney Diseases, 15, 601-602. http://dx.doi.org/10.1016/S0272-6386(12)80534-8

[11] Anwar, N. and Gokal, R. (1993) Simultaneous Occurrence of IgA Nephropathy and Sarcoidosis in the Context of PreExistent Minimal Change Nephrotic Syndrome. Nephron, 65, 310-312. http://dx.doi.org/10.1159/000187494

[12] Tateno, S., Kobayashi, Y. and Kobayashi, F. (1994) A Case of Sarcoidosis Revealed in the Course of IgA Nephropathy. Pathology International, 44, 387-390. http://dx.doi.org/10.1111/j.1440-1827.1994.tb02939.x

[13] Nishiya, H., Yoshida, H., Tomonari, H., Hikita, M., Shike, T., Takeda, Y., et al. (1996) Sarcoidosis Representing Multiple Splenic Nodules in a Patient with IgA Nephropathy. Nihon Jinzo Gakkai Shi, 38, 40-45.

[14] Hamada, K., Nagai, S., Ono, T., Muso, E., Nagao, T., Shigematsu, M., et al. (2003) Sarcoidosis Complicated with IgA Nephropathy. Sarcoidosis, Vasculitis and Diffuse Lung Diseases, 20, 69-73.

[15] Kahn, A., Hodges, N. and Lord, M. (2005) A Case of Sarcoidosis in a Patient with IgA Nephropathy. Medscape General Medicine, 7, 7.

[16] Taylor, J.E. and Ansell, I.D. (1996) Steroid-Sensitive Nephrotic Syndrome and Renal Impairment in a Patient with Sarcoidosis and IgA Nephropathy. Nephrology Dialysis Transplantation, 11, 355-356. http://dx.doi.org/10.1093/oxfordjournals.ndt.a027267 